\title{
Gestión y evaluación del rendimiento en baloncesto: una revisión sistemática del software Management and performance analysis in basketball: a systematic review of software
}

\author{
Álvaro Bustamante, Pablo Burillo \\ Universidad Camilo José Cela (España)
}

Resumen. Introducción: La gestión de los recursos y el análisis del rendimiento son determinantes en cualquier entidad deportiva. En el caso de deportes de equipo como el baloncesto, este tipo de herramientas son básicas para ayudar en la toma de decisiones. Objetivos: Realizar una revisión bibliográfica sobre las tendencias actuales en el desarrollo de sistemas informáticos de gestión deportiva y análisis del rendimiento. Y revisar las herramientas informáticas que en la actualidad prestan este tipo de servicios. Método: Se realizó una búsqueda bibliográfica exhaustiva en cinco bases de datos de referencia en investigación deportiva, obteniendo 717 resultados de los cuales se consideraron relevantes un total de 58 . Además, se realizó una búsqueda electrónica de herramientas informáticas de referencia en el sector deportivo y se han analizado 22 de ellas, estudiando sus características, autores, presupuesto y limitaciones. Resultados: Existen características comunes en el desarrollo de herramientas de gestión y análisis del rendimiento deportivo: acceso web, calendario de planificación, registro de la evolución de los jugadores, repositorios de ejercicios y gestión económica, entre otras. Conclusiones: Tradicionalmente no se han integrado las herramientas de gestión y análisis del rendimiento en baloncesto, pero sí existe software que proporcione ambos servicios en fútbol. Los estudios futuros versarán sobre cuestiones de privacidad de datos y la mejora de los sistemas expertos. Palabras clave: software, gestión, club, análisis, baloncesto.

Abstract. Background: Human resources management and performance analysis are decisive for any sport institution. Moreover, sport managers being in charge of sport clubs must tackle specific issues related to the sport sector. For team sports like basketball, these kind of tools are very important to help in analysis and decision making. Objectives: To find current trends in sport management and performance analysis software. In addition» 0 «Additionally, to review actual management and performance software tools. Method: An systematic scientific literature research has been made for five sport-specific research databases. 717 results were gathered and 58 of them were relevant to this study. Some further electronic research was made in order to find valuable software tools for this field. 22 of them were revised taking into account their features, authors, budget and limitations. Results: Common features have been identified for the development of management and performance analysis of sports. Some common features for this kind of software are: web access, planning calendar, player evolution follow-up, exercise databases and economic management, among others. Conclusions: Traditionally, management and performance analysis have not been integrated in one tool for basketball, but some new software is being designed for supporting both in football (soccer). Future studies will tackle data privacy and the improvement of the use of expert systems.

Keywords: software, management, club, analysis, basketball.

\section{Introducción}

Los recursos humanos, así como la capacidad de desarrollo y planificación se han identificado como factores de importancia en la consecución de objetivos en organizaciones deportivas (Misener \& Doherty, 2009). Más allá de la existencia de esta necesidad, los recursos humanos son críticos para la efectividad de las entidades y por ello su gestión debe ser eficaz. El éxito de la gestión depende de la habilidad de explicar y predecir el comportamiento de la organización, es decir, de dirigir las actitudes y comportamientos de individuos y grupos de la entidad (Doherty, 1998).

Para hacer frente a los retos y necesidades actuales, cualquier organización de gestión deportiva debe asegurar que su modelo interno sea de alta calidad, lo que ayudará en promover un óptimo funcionamiento operativo (Cano \& Cano, 2006).

Además, el ámbito deportivo requiere la realización de tareas complejas por parte de los managers. Esta complejidad se acentúa aún más por el hecho de que dichas tareas incluyen la gestión de cuestiones no sólo deportivas, sino que también incluyen aspectos auxiliares tales como: equipamiento, mantenimiento de instalaciones, gestión de empleados y usuarios (Gallardo-Guerrero, Garcia-Tascon, \& Burillo-Naranjo, 2008).

Con el rápido desarrollo de la industria del deporte, los métodos tradicionales de gestión del deporteno son tan adecuados en la sociedad moderna. Actualmente, las tecnologías de la información pueden mejorar el trabajo y la eficiencia de la gestión de manera significativa (Li \& Wang, 2012).

Los sistemas de información desarrollados para la gestión han sido utilizados en diversos ámbitos relacionados con el deporte, tales como la personalización ante la diversidad de discapacidades que presenta el deporte adaptado (Marcelo \& Oltra, 2005), la potenciación de relaciones de satisfacción y lealtad con los aficionados mediante las páginas web de los clubes deportivos (Hur, Ko, \& Valacich, 2011) o la

Fecha recepción: 22-10-14- Fecha envío revisores: 24-11-14- Fecha de aceptación: 10-08-15 Álvaro Bustamante busta.es@gmail.com monitorización de lesiones deportivas (Hanisch, Hanisch, \& Pacis, 2007).

El software ideal para los managers deportivos debería permitir fácil acceso a varias bases de datos y sistemas de información. También debería ser modular, debido a la gran variedad de instalaciones deportivas, perfiles variados de gestores y la enorme cantidad de factores a considerar al gestionar una instalación de tipo deportivo (GallardoGuerrero et al., 2008).

En el ámbito del rendimiento deportivo, también existe una creciente necesidad de colección de datos que aporten evidencia empírica sobre la compleja realidad a la que se refieren. Existe una carencia de instrumentos estándar de observación y una necesidad de desarrollar sistemas de codificación por ordenador adecuados para estos contextos (Fernandez, Camerino, Anguera, \& Jonsson, 2009).

En el caso de deportes de equipo como el baloncesto, existe una necesidad de gestionar el conocimiento, los sistemas de logística y la comunicación en los clubes de manera correcta (Orekovic, 2007). Además, se ha notado un visible progreso en el análisis de los equipos y jugadores rivales. El profesionalismo hace que los clubes afronten sus actividades de manera cada vez más sistemática y utilicen programas informáticos para gestionar tácticas, informes y vídeos (Markoski, Pecev, Ivkovic, Ivankovic, \& Ratgeber, 2012).

La capacidad de producir datos para el análisis del rendimiento deportivo ha sido mejorada en la última década debido a avances tecnológicos sustanciales (Liebermann etal., 2002; Nevill,Atkinson, \& Hughes, 2008). Sin embargo, los métodos actuales de análisis del rendimiento han sido criticados por carencias de infraestructuras que regulen la evaluación del rendimiento. Por lo tanto, todavía no es suficientemente claro cómo un entrenador podría demostrar un rendimiento satisfactorio durante la competición (Travassos, Davids, Araujo, \& Esteves, 2013).

Esta diversidad de perfiles, instalaciones y factores invita a pensar que la mayoría de herramientas son orientadas a dominios específicos. Y aún existen carencias de herramientas con capacidad de gestionar instalaciones, empleados, atletas y equipos en el ámbito de una entidad deportiva.

La presente revisión tiene como objetivo principal realizar un análisis de herramientas tecnológicas de gestión de clubes deportivos. Ade- 
más, se plantean los siguientes objetivos secundarios: 1) localizar herramientas tecnológicas que integren tanto la gestión como la evaluación del rendimiento en clubes deportivos de baloncesto y 2) revisar la literatura científica para analizar las últimas tendencias en el desarrollo de herramientas tecnológicas de gestión y evaluación del rendimiento deportivo.

\section{Método}

La búsqueda bibliográfica se realizó de manera electrónica durante los meses de Abril y Mayo de 2014, utilizando las siguientes bases de datos:

Web of Science
EBSCOHost
SCOPUS
Dialnet
Teseo

A continuación, se especifican los descriptores utilizados para realizar las búsquedas.

Descriptores en inglés

- Basketball management software

Basketball club management

Sport club software

Sport training management technology

- Basketball video analysis software

Descriptores en español

- Software de gestión en baloncesto

- Gestión de clubes de baloncesto

- Software para clubes deportivos

- Tecnologías para la gestión del entrenamiento deportivo

- Software de análisis de vídeo en baloncesto

Las restricciones empleadas fueron las siguientes: 1) el idioma en el que estaban escritos los artículos debía ser inglés o español, 2) los artículos debían estar publicados en el periodo comprendido entre el año 2000 y el año 2014, y 3) tratar sobre el uso de tecnologías orientadas a mejorar la gestión del entrenamiento de equipos deportivos.

Se obtuvieron un total de 717 resultados, de los cuales 541 eran artículos y 16 revisiones. El resto de resultados correspondieron a patentes, meetings y otro tipo de documentos.

Estos resultados se filtraron conforme a los tres criterios citados anteriormente hasta llegar a un total de 58 artículos válidos, entre los cuales existen dos revisiones y ninguna tesis doctoral.

Además de realizar una búsqueda de artículos de investigación, se realizó una búsqueda de herramientas tecnológicas de gestión de equipos deportivos. Esta búsqueda se llevó a cabo de manera electrónica en internet y pretende aportar a la revisión un resumen de características de las herramientas tecnológicas ya implementadas en la actualidad.

Se encontraron un total de 45 herramientas tecnológicas de gestión de equipos deportivos. Cumplieron los siguientes requisitos de inclusión: 1) tratar sobre la gestión del entrenamiento en clubes deportivos y 2) ser operativas en la actualidad, un total de 22 herramientas.

\section{Estado de la cuestión y revisión de herramientas}

\section{Estado de la cuestión}

El uso de las nuevas tecnologías para evaluar y dar feedback ocurre en contextos cada vez más exigentes, y se necesita entender cómo alcanzar los mejores resultados de la manera más eficiente (JISC, 2014). En este sentido, las organizaciones necesitan ser conscientes de la necesidad de nuevos sistemas de información para seguir teniendo éxito, calidad y excelencia en la gestión (Senlle, Gallardo, \& Dorado, 2004). En definitiva: los gestores de organizaciones deportivas tienen la necesidad de usar programas informáticos específicamente diseñados para sus funciones (Gallardo-Guerrero et al., 2008).

Esta necesidad se hace también evidente en el ámbito puramente deportivo. La importancia de un buen feedback en el proceso de adquisición de habilidades motoras ha sido objeto de estudio por diversos autores (Schmidt \& Lee, 1999) y existen evidencias de que una buena comunicación con el deportista es un factor decisivo en la mejora de aptitudes deportivas (Liebermann et al., 2002).

Tradicionalmente, se han detectado necesidades de analizar el rendimiento deportivo de manera sistemática y racional (Franks \& Goodman, 1986), de tal manera que los entrenadores tengan datos objetivos que transmitir en el proceso de feedback entre entrenador y deportista (Nevill et al., 2008). Actualmente se están desarrollando soluciones tecnológicas capaces de recoger datos de los deportistas para usar esa información y mejorar su rendimiento en eventos como los Juegos Olímpicos (Mertz, 2013),

Esta preferencia ha contribuido a que se investigue sobre el desarrollo de aplicaciones de gestión y análisis del rendimiento. En una de estas investigaciones se defiende el uso de sistemas cliente-servidor y el diseño de una base de datos que permita a los entrenadores gestionar la evolución de sus atletas (Simin \& Jinhai, 2009).

La inclinación a almacenar los datos en un servidor coincide con otra investigación que defiende el papel de las bases de datos y los sistemas expertos para mejorar la eficiencia de la gestión deportiva (Li \& Wang, 2012). El desarrollo web, unido a los sistemas inteligentes también se ha utilizado para facilitar el desarrollo de algoritmos de predicción de resultados (Mateos, 2011).

El hecho de que el rendimiento deportivo se vea afectado por numerosas variables difíciles de analizar, ha propiciado que se haya investigado la manera de tratar con datos biológicos y cinemáticos mediante cloud computing (Wu, Zhao, \& Zhao, 2013).

En este sentido, sigue habiendo cierta confusión entre atletas y entrenadores a la hora de utilizar este tipo de sistemas, por lo que hay una clara necesidad de estandarización en las técnicas de medida, protocolos de transmisión y estructuración de datos (Baca, Dabnichki, Heller, \& Kornfeind, 2009), Además, estos sistemas deben afrontar cuestiones éticas debido a la compartición de datos personales de los deportistas (Baca et al., 2009).

El análisis notacional es una manera objetiva de registrar el rendimiento de tal manera que los elementos claves puedan ser cuantificados de manera consistente y válida (Nevill et al., 2008). Antes de que se utilizara el análisis notacional, algunos autores llegaron a la conclusión de que los atletas desarrollan un comportamiento observable que puede ser cuantificado y, en última instancia, modificado (Franks \& Goodman, 1986). Siguiendo esta línea de investigación, se llegó a la conclusión de que estos aspectos de comportamiento efectivamente pueden ser registrados mediante análisis notacional y que su aplicación podría mejorar los siguientes aspectos: 1) evaluación táctica, 2) evaluación técnica y 3) análisis de movimientos (Hughes \& Franks, 2004).

A pesar de la ayuda que representa contar con estas herramientas, el análisis del rendimiento actual tiende a centrarse en variables cuantitativas individuales. Recientes investigaciones sobre dinámicas ecológicas defienden que los analistas deberían combinar la descripción de estas variables con relaciones espacio-temporales entre los jugadores para mejorar los sistemas de entrenamiento (Travassos et al., 2013).

La tendencia futura en el uso de sistemas inteligentes se centrará no sólo en la propia tecnología sino en el uso de redes sociales más extensas que impulsen la interacción y diseminación del conocimiento empírico y científico (Baca et al., 2009).

\section{Herramientas tecnológicas}

En este apartado se van a comentar las características principales de diversas herramientas tecnológicas orientadas al análisis y la gestión de equipos deportivos. En los siguientes apartados, se presentan 5 subapartados para el análisis de cada herramienta: definición, servicios, autor, presupuesto y limitaciones de cara al objetivo especificado en el presente artículo.

\section{DVSportFastbreak}

Definición: Solución software de análisis de video, hecha a medida, diseñada mediante el uso de estándares industriales y feedback directo con entrenadores, expertos en vídeo y jugadores.

Es una herramienta personalizable y escalable que se ajusta las 
necesidades y el presupuesto de cualquier equipo de baloncesto. Es proveedor de diversos equipos profesionales y universitarios de baloncesto, fútbol americano, hockey y lacrosse.

\section{Servicios:}

o Captura vídeo desde broadcast feed o fuentes de internet

o Interfaz personalizable

o Controlador con puntero láser

o Posibilidad de subir y acceder a fragmentos en la nube

o Personalización de datos y generación de gráficos e informes

o Frame by frame slow motion, telestrator y soporte de múltiples monitores

o Integración con PowerPoint, combinando presentación y fragmentos

Autores: DVSport Inc. (DVSport, 2014)

Presupuesto: Personalizado dependiendo de las necesidades de cada club.

Limitaciones: No presenta herramientas de gestión y seguimiento del entrenamiento.

\section{Kinovea}

Definición: Es un reproductor y editor de vídeo para analizar, estudiar y comentar la técnica de los atletas. El editor ofrece herramientas para estudiar los movimientos mediante la creación de telestrators, comparación de secuencias, etc.

Servicios:

o Medición de distancias y ángulos

o Interfaz simple pero con muchas posibilidades

o Telestrator

o Comparación de vídeos simultáneos

o Solución de código abierto

Autores: Kinovea (Kinovea, 2014)

Presupuesto: Gratuita.

Limitaciones: No presenta herramientas de gestión y seguimiento del entrenamiento y necesita instalación en el dispositivo (no proporciona acceso web).

\section{Hudl}

Definición: Hudl es una herramienta dedicada al análisis de vídeo, ofreciendo servicios tanto a entrenadores como a deportistas y ojeadores. La herramienta tiene una interfaz simple y proporciona acceso gratuito a apps Android, iPhone y iPad con su licencia. También proporciona acceso a videos compartidos.

Soporta varios deportes: fútbol americano, voleibol, baloncesto, lucha, atletismo, lacrosse. Actualmente cuenta con tres millones de usuarios y 20 equipos profesionales.

Servicios:

o Filtrado de partidos por posesiones

o Permite añadir: telestrators, notas y compartir con entrenadores y jugadores

o App móvil para ver playlists en la nube

o Etiquetado con terminología personalizada, para obtener playlists más simples

o Almacenamiento de partidos en la nube

o Cámara lenta, mensajería, informes detallados en PDF

o Pone en contacto: entrenadores, atletas y ojeadores con su sistema de intercambio de vídeos

Autores: Hudl (Hudl, 2014)

Presupuesto:

o High school: \$800-3.000/año

o Coach: \$99-299/año

o Athlete: \$200/año

o Equipos profesionales y clubes: pedir presupuesto personalizado

Limitaciones: No presenta herramientas de gestión y seguimiento del entrenamiento y necesita instalación en el dispositivo (no proporciona acceso web).

\section{SoccerLAB}

Definición: SoccerLAB es un sistema centralizado para apoyar al personal técnico de un club de fútbol o federación. Combina varias aplicaciones de software (cliente y servidor), hardware y servicios de mantenimiento. SoccerLAB es una aplicación web, por lo que es accesible desde cualquier navegador sin necesidad de instalar aplicaciones. Un sistema de administración de usuarios y seguridad de gran alcance hace posible que los usuarios puedan trabajar juntos en la misma base de datos. Al establecer los privilegios correctos los usuarios sólo pueden ver lo que se les permite ver. Cada departamento y unidad dentro de la organización pueden trabajar de una manera segura en sus propios datos sensibles.

\section{Servicios:}

o Módulos principales de datos: scouting, formación de equipo, calendario, médico y rendimiento

oAnálisis de video en línea

o Multiusuario, con normas de seguridad y autorización

o Posibilidad de asociar metadatos (nombre, descripción, etiquetas) a los clips de vídeo

o Compartir clips de vídeo con los jugadores y entrenadores

o Función de chat

o Múltiples formatos de vídeo compatibles

o Sharing Clip móvil para tablet (iPad o Android) para ver los clips y comentarios subidos por el entrenador

o Herramienta de presentación de diapositivas

o Herramientas de telestrator

Autores: SoccerLab(SoccerLAB, 2014)

Presupuesto: Pedir presupuesto, previo registro en la página web. Limitaciones: Aplicación específica para fútbol.

\section{Nacsport}

Definición: Este software está dirigido a entrenadores de equipos de todos los niveles y una de sus mayores ventajas es la personalización de etiquetas con las que catalogar las acciones que se crean pertinentes.

Los tutoriales de su página web explican la manera de trabajar con Nacsport en 3 pasos: 1) toma de datos, 2) trabajo de datos, 3) presentación del trabajo.

\section{Servicios:}

o Timeline con las acciones registradas.

o Posibilidad de imprimir o exportar informes a Excel o PDF

o Personalización de categorías, insertar notas de texto y telestrator o Comparar hasta 8 acciones

o Playlist de acciones

o Organización de entrenamientos y partidos con librería de ejercicios

Autores: Nacsport (Nacsport, 2014)

Presupuesto:

o Basic (150 euros, I.V.A. no incluido)

o Basic plus (550 euros, I.V.A. no incluido)

o Scout plus (1200 euros, I.V.A. no incluido)

o Pro plus (pedir presupuesto)

o Elite (pedir presupuesto)

Limitaciones: Aunque presenta un módulo dedicado a la planificación, no presenta bases de datos de seguimiento de jugadores.

\section{Espor}

Definición: Espor es un editor de vídeo desarrollado específicamente para el análisis deportivo. Ofrece un servicio de codificación que hace que los entrenadores no tengan que hacer manualmente la fragmentación de las acciones. Está disponible para diferentes deportes tales como: baloncesto, fútbol, futbol americano, lacrosse, hockey y vóley.

Servicios:

oPermite el streaming de tus partidos y el etiquetado en el momen-
o. Permite importar y exportar múltiples formatos y compartirlos
Retos, número 29, 2016 ( $1^{\circ}$ semestre) 
en la nube de manera instantánea.

o Exportación de informes, cartas de tiro.

o Telestrator, imágenes y formato de diapositivas.

Autores: Espor, Inc. (Espor, 2014)

Presupuesto: Es necesario pedir presupuesto personalizado.

Limitaciones: No presenta herramientas de gestión y seguimiento del entrenamiento

\section{SPORTSTEC}

Definición: Este software está designado de manera exclusiva para equipos e individuos que desarrollan su actividad en torno al rendimiento deportivo. Posibilita analizar y revisar vídeo y datos de partidos de manera rápida y fácil. Módulos:

o Sportscode elite y pro.

o Gamebreaker y gamebreaker+

o Sportscode review

o Track (patrones de movimiento)

o CODA

o Sportscode player

o Sporstec EXCHANGE y STREAM

Servicios:

o Captura de vídeo: captura de vídeo en HD

o Codificación: posibilidad de etiquetar las acciones relevantes para el rendimiento de tu equipo

o Revisión: proporciona funciones de búsqueda inteligente y detallada para reunir evidencias visuales y repeticiones de patrones seleccionados. También dispone de etiquetados que proporcionan playlists adecuadas

o Compartición: Proporciona acceso a la nube de todo tu equipo y acceso a la información en cualquier momento

Autores: Sportstec (Sportstec, 2014)

\section{Presupuesto:}

o Plus: \$3.200/año

o Standard: \$1600/año

o Basic: \$800/ año

Limitaciones: No presenta herramientas de gestión y seguimiento del entrenamiento.

\section{Vidswap}

Definición: Vidswap es una herramienta online que se encarga de etiquetar cualquier vídeo específicamente para cada uno de los deportes que soporta (fútbol, fútbol americano, hockey, baloncesto, lacrosse, vóley, béisbol, sóftbol y rugby). Lo único que el usuario debe hacer es grabarlo, subirlo, y en menos de 24 horas puede consultarlo en la web. El equipo de vidswap proporciona las estadísticas propias de cada deporte.

\section{Servicios:}

o Análisis por jugadas y por timeline

o Acceso a la nube con iPad y iPhone

o Estadísticas e informes personalizados

o Datos personalizados

o Teclas de acceso directo y telestrator

o Creación de cuentas para tu equipo (jugadores y entrenadores)

o Ángulos múltiples

o Mensajería de vídeo e intercambio de vídeo con jugadores y entrenadores

Autores: Vidswap (Vidswap, 2014)

Presupuesto:

o Plus: \$3200/año

o Standard: \$1600/ año

\section{Skaut}

Definición: Skaut es una herramienta elaborada para que los técnicos y entrenadores deportivos puedan diseñar ejercicios de carácter técnico, físico, táctico y/o estratégico de una manera organizada y clasificada a modo de cuaderno de entrenamiento.
Permite crear actividades y/o ejercicios de entrenamiento, en uno o varios cuadernos digitales específicos.

Los técnicos deportivos con la que podrán crear simulaciones que favorezcan el proceso de enseñanza-aprendizaje, la comunicación con los deportistas y la trasmisión de conocimientos.

Licencias: Analyst TOTAL, Analyst COMPACT, TotalStation, Viewer, Pocket, Plan, TactLab.

Servicios:

o Módulo de clasificación de contenidos.

o Módulo de diseño de ejercicios

o Módulo de búsqueda. Permite buscar aquellos ejercicios de tus cuadernos tácticos digitales que cumplan con unas determinadas características

o Módulo de creación de informe: elaboración de informes de sesión de entrenamiento

o Módulo de visualización: ofrece visionar los ejercicios en pantalla completa y utilizar la aplicación a modo de pizarra táctica digital.

o Deportes: baloncesto, balonmano, fútbol, futbol sala, hockey, tenis, voleibol y pádel

Autores: Skaut Technology\&consulting (Skaut, 2014)

Presupuesto: Pedir presupuesto personalizado.

Limitaciones: Aunque tiene módulos de análisis de vídeo y de creación de ejercicios, aún no se ha desarrollado un módulo de planificación de la temporada y de integración de dichos módulos.

\section{Dartfish}

Descripción: Dartfish integra software y herramientas en línea para ofrecer un sistema de análisis de deportistas flexible, eficiente y completo.

La solución Dartfish Tagging hace que sea fácil encontrar, revisar y analizar las secuencias fragmentos en un partido. Éstos están marcados usando «etiquetas» descriptivas que pueden integrarse de manera personalizada mediante EasyTag.

Las etiquetas incluso pueden ser importados de otro software o añadidas a partir de dartfish.tv. También proporciona estadísticas instantáneas una vez hecho el etiquetado.

\section{Servicios:}

o Dartfish TeamPro y Connect Plus: software de análisis de vídeo o Dartfish EasyTag: aplicación para los dispositivos iPhone , iPad

y Android

o Dartfish.tv (plataforma de vídeo online)

o Biblioteca de vídeos

o Visualización de puntos estratégicos y tácticos

o Compartir contenido con los jugadores, entrenadores

o Acceso a fragmentos en la nube

o Solución flexible para cualquier deporte

o Editor de notas y correo electrónico

Autores: Dartfish (Dartfish, 2014)

Presupuesto: Pedir presupuesto personalizado.

Limitaciones: No presenta herramientas de gestión y seguimiento del entrenamiento.

\section{E-ntrenamiento}

Definición: E-ntrenamiento es un software deportivo on-line, un programa informático para diseñar entrenamientos y ejercicios, para ayudarte en la confección de las sesiones de entrenamiento de tus equipos de futbol, de baloncesto o de balonmano.

\section{Servicios:}

o Listado de entrenamientos, posibilidad de introducir nuevos ejer-

cicios

o Base de datos creada por entrenadores cualificados con 1062 ejercicios

o Resultados y estadísticas

o Fichas de cada jugador para llevar un control de cada uno de ellos

Autores: Dartfish (Dartfish, 2014)

Presupuesto: Pedir presupuesto personalizado

Limitaciones: No soporta análisis de vídeo. 


\section{Mundomonitor}

Definición: Plataforma web gratuita, que permite a los entrenadores gestionar equipos. Incorpora video tutoriales para ayudar al usuario y es necesario un registro web previo a su utilización.

Además da acceso a una comunidad de usuarios (previo pago) con la que se puede compartir y acceder a ejercicios ya realizados y permite su importación automática.

Durante el año 2014 se está desarrollando una herramienta para coordinadores de entrenadores que permitiría la gestión de responsabilidades y objetivos.

Servicios:

o Calendario, equipos, temporadas, sesiones de entrenamiento y ejercicios

o Personalización de categorías

o Estadísticas individuales y colectivas

o Competiciones

o Staff técnico

o Gestión de informes

o Libreta de contactos y mailing

o Soporta los siguientes deportes: fútbol, fútbol sala, fútbol 7, baloncesto, balonmano, hockey hielo, hockey patines, hockey hierba, rugby, fútbol americano, voleibol y waterpolo

Autores: Mundomonitor.com (Mundomonitor.com, 2014)

Presupuesto: Acceso a la comunidad: 12 euros/año.

Limitaciones: Herramienta muy completa, pero que no soporta análisis de vídeo y presenta problemas con algunos navegadores.

\section{GESDEP}

Definición: Solución integrada para la gestión de entidades deportivas, sin necesidad de instalaciones en el ordenador y accesible desde cualquier punto donde exista una conexión a internet. 15 años de experiencia en software orientado al deporte avalan a este producto, fruto de la combinación de profesionales informáticos con especialistas en la gestión y el entrenamiento deportivo.

\section{Servicios:}

o Fichas de jugadores, estadísticas y expedientes disciplinarios

o Presupuestos del club y pagos a jugadores

o Gestión de socios y padres de jugadores

o Informes de partidos, calendario, partidos, equipos

o Base de datos de ejercicios

o Planificación de entrenamientos

o Mensajería interna

Autores: Gesdep.net (Gesdep.net, 2014)

Presupuesto:

o Entrenador: 55 euros/año

o Club: 265 euros/año

o Club+: 365 euros/año

Limitaciones: No soporta análisis de vídeo.

\section{Rincoach}

Definición: Herramienta web para entrenadores de fútbol, creada en forma de red de entrenadores que ofrece herramientas de gestión, planificación y ejercicios ampliamente personalizables. Existe una versión gratuita y versiones mejoradas de pago.

\section{Servicios:}

o Herramienta de gestión de entrenamientos

o Planificación deportiva

o Ejercicios personalizados

o Importación de ejercicios desde la comunidad

o Aplicación para el seguimiento de jugadores y equipos

Autores: Sportsulting (Rincoach, 2014)

\section{Presupuesto:}

o Free (gratuita)

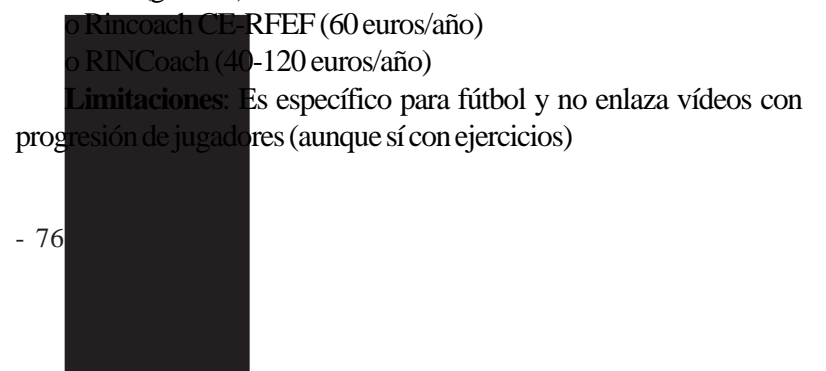

\section{Campusdeportivo}

Definición: Herramienta informática para entrenadores que sirve para el diseño y archivo de ejercicios y sesiones de entrenamiento, situaciones tácticas, jugadas de estrategia, etc. Ayuda a planificar sesiones de entrenamiento mediante ejercicios que se pueden modificar e imprimir.

Servicios:

o Diseño de sesiones de entreno

o Diseño de ejercicios

o Modificación de ejercicios y gráficos personalizables

o Deportes: fútbol, baloncesto, balonmano, fútbol sala, etc.

o Válido para Windows

2014)

Autores: Campusdeportivovirtual (Campusdeportivovirtual,

Presupuesto: 14,90 euros/descarga.

Limitaciones: No proporciona acceso web y no soporta análisis de vídeo.

\section{Kimetplanning}

Definición: Kimet Planning es una aplicación informática de fútbol con «inteligencia propia» para planificar la temporada cualquier equipo.

Para ello, la aplicación proporciona una metodología estándar (contrastada por los autores de la aplicación) o bien que el usuario elija sus propias preferencias en base a las que el sistema calculará las sesiones de entrenamiento, cargas y ejercicios más adecuados.

Servicios:

o Planificación inteligente en 5 pasos

o Adaptado al nivel y categoría elegida

o Personalización con criterios propios

o Base de datos de ejercicios (más de mil)

oAnimador gráfico de ejercicios

o Mecanismos de control: asistencia, estadísticas, lesiones, cargas físicas

o Informes y estadísticas

o Evaluación física, técnica y táctica

o Tareas de gestión: calendario, autobuses, gestión de campos

Autores: Kimetsport (Kimetplanning, 2014)

Presupuesto:

o Coach: 49,90 euros/año

o Team: 99 euros/año

o Club: presupuesto personalizado

Limitaciones: Específico para fútbol. No soporta análisis de vídeo para incorporar a la evaluación de jugadores.

\section{Procloud11}

Definición: Aplicación que recoge todas las funciones y trabajos que se desempeñan dentro de un cuerpo técnico de fútbol. Su carácter multi-usuario permite que todos los miembros del cuerpo técnico puedan trabajar de forma simultánea.

Los entrenadores pueden almacenar y guardar todos los ejercicios en una base de datos de fácil acceso. El sistema ProCloud11 ofrece la posibilidad de ver el ejercicio en cualquier momento, cuándo ha sido utilizado por última vez y con qué frecuencia, las valoraciones que se han hecho del ejercicio y sus posibles variantes.

La aplicación permite trabajar con ella a distancia, gracias a la tecnología Cloud.

Servicios:

o Estudiar el funcionamiento del equipo con estadísticas: resultados, clasificación y tendencias de equipos

o Planificación y contenidos entrenamientos

o Perfiles psicológicos, lesiones y datos personales

o Gestión de grupos de trabajo para que todos los miembros del equipo técnico trabajen en sus áreas

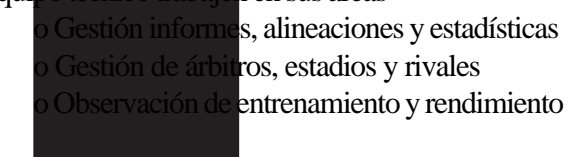


o Sistema de mensajería interna

oAcceso cloud

Autores: Procloud11 (Procloud11, 2014)

Presupuesto:

o Scout\&agent: 50 euros

o Scout\&agent pro: 299 euros

o Entrenador: 69 euros

o Cuerpo técnico: 399 euros

Limitaciones: No soporta análisis de vídeo para evaluar jugadores. Programa específico para fútbol.

\section{Easy2coach}

Definición: Herramienta que permite la planificación de entrenamientos y partidos de fútbol desde niveles amateur a profesional.

Incluye módulos de estadísticas, ejercicios, tácticas y favorece la comunicación entre los miembros del equipo.

Servicios:

o Planificación y organización de equipos

o Valoraciones automáticas y estadísticas detalladas

o Bases de datos de ejercicios con herramientas de búsqueda y animaciones.

o Gestión de equipos de categorías inferiores

Autores: Easy2coach (Easy2Coach, 2014

Presupuesto: 359 euros/año.

Limitaciones: Específica para fútbol y no incluye módulo de análisis de vídeo.

\section{Interplay-sports}

Definición: Esta herramienta de análisis de partidos presenta tres tipos de licencias: licencia simple (para un solo ordenador), licencia de club (para tres ordenadores, además de 7 licencias para entrenadores y jugadores) y paquete de federación (que incluye licencia para todos los entrenadores a nivel nacional)

Módulos: Pro, semiPro y miniPro.

Deportes: fútbol, hockey sobre hielo, tenis, baloncesto, Balonmano, rugby, lacrosse, fútbol sala, fútbol, balonmano.

\section{Servicios:}

o Permite dibujar posiciones y acciones de juego

o Teclas de acceso rápido

o Creación de categorías y grupos a varios niveles y de forma personalizada

o Etiquetado de jugadores

o Conexión con cámaras

o Creación de grupos de trabajo

o Análisis en vivo y con resultados e mitad de partido mediante tecnología WiFi

Autores: Interplay-sports (Interplay-sports, 2014)

Presupuesto: Pedir presupuesto personalizado.

Limitaciones: No presenta herramientas de gestión y seguimiento del entrenamiento.

\section{NovaNET}

Definición: Sistema integral de Gestión que permite que clubes deportivos, de cualquier tamaño, puedan gestionar todas las actividades, administrativas y deportivas, de una manera sencilla.

Esta solución integra todas las áreas del Club a la vez de proporcionar las herramientas necesarias para mejorar tanto el área deportiva como el área económica del Club.

El sistema está desarrollado siguiendo los criterios basados en la Web 2.0 .

Tiene módulos de gestión de clubes, comité de entrenadores, escuela de entrenadores, gestión deportiva, gestión selecciones, competiciones y entradas, además de tv por internet y red social.

Servicios:

o Gestión de Tesorería, control de proveedores, elaboración de presupuestos e informes económicos

o Ficha completa de jugadores y técnicos, estadísticas o Newsletter del club, correo interno, agenda, horarios, desplazamientos

o Preparación de entrenamientos, creación de fichas de entrenamientos, pizarra electrónica

o Repositorio de ejercicios

o Análisis de rendimiento, planificación de equipos, horarios, desplazamientos, etc.,

o Gestión y reserva de campos

o Gestión y venta de entradas y mantenimiento de socios

Autores: NOVANET AUTOMATION CONSULTANTS SL (NovaNET, 2014)

Presupuesto: Pedir presupuesto personalizado.

Limitaciones: No soporta análisis de vídeo para evaluar jugadores.

Sport-Designer

Definición: Herramienta de diseño y gestión multideportiva orientada al diseño de ejercicios. Incluye 14 disciplinas deportivas, más de 10 idiomas, contenidos on-line y acceso a versión móvil.

Servicios:

o Paleta de herramientas para diseñar ejercicios

o Múltiples plantillas para diferentes deportes

o Animaciones de situaciones de juego

o Creación de jugadas de estrategia y formatos de convocatorias

o Libros de ejercicios y estrategia

Autores: Grupo-campus (Grupo-campus, 2014)

Presupuesto: 42,85 euros

Limitaciones: Es específica para diseñar ejercicios y no presenta módulos de gestión de la temporada ni soporta análisis de vídeo.

Diamond Scheduler

Definición: Diamond Scheduler es un programa para Windows que permite crear calendarios para diversos deportes: béisbol, softball, fútbol, baloncesto, etc. Permite exportar horarios a sitios web y cuenta con más de 20 configuraciones de uso.

Servicios:

o Personalización de equipos y jugadores

o Personalización de categorías y campos de juego

o Personalización de normas de los torneos con calendario incorpo-

rado

o Emparejamientos y eliminatorias automáticas tras fases de grupos

Autores: Cactusware(DiamondScheduler, 2014)

Presupuesto: \$70-75

Limitaciones: Únicamente incluye gestión de torneos; no incluye análisis de vídeo ni planificación del entrenamiento.

En la Tabla 1 se muestra una agrupación de las herramientas analizadas, siguiendo dos criterios: 1) si soportan baloncesto, y 2) si soportan análisis de rendimiento o gestión y planificación.

Se puede observar que sólo existen dos herramientas que soportan en análisis del rendimiento y la planificación de entidades (SoccerLAB y Skaut) y ambas están fuera del dominio del baloncesto al ser específicas para fútbol.

Tabla 1

\begin{tabular}{ccc} 
Categorización de las herramientas tecnológicas & \\
\hline \multicolumn{1}{c}{ Categorización } & $\begin{array}{c}\text { Análisis de vídeo y } \\
\text { rendimiento }\end{array}$ & G estión y planificación \\
\hline & DVSport & Mundomonitor \\
& Hudl & Gesdep \\
& Sportstec & NovaNet \\
1. Soporta baloncesto & Nacsport & Campus deportivo \\
& Kinovea & Sport-designer \\
& Interplay-sports & Diamond-scheduler \\
& Vidswap & E-ntrenamiento \\
& Espor & SoccerLAB \\
& Dartfish & Skaut \\
& & KimmetPlanning \\
2. No soporta baloncesto & Procloud11 \\
& SoccerLAB & Easy2coach \\
& Skaut & Rincoach \\
\hline
\end{tabular}


La revisión conjunta de la literatura científica y de las herramientas actuales, ha hecho posible la síntesis de los aspectos clave en el diseño de este tipo de herramientas:

1) Uso de sistemas de información web y disponibilidad de contenidos mediante acceso a internet.

2) Seguimiento y evaluación de jugadores y equipos, con generación de informes personalizados mediante sistemas expertos.

3) Disposición de un calendario de planificación.

4) Acceso a repositorios de ejercicios.

5) Categorización personalizada y etiquetado de contenidos.

6) Gestión de normas de acceso a contenidos para cada tipo de usuario: jugador, entrenador y otro tipo de usuario (padres, agentes, ojeadores, etc.).

7) Servicios de mensajería interna y/o chat.

8) Evaluación y planificación económica de la institución.

9) Subproductos con escalas presupuestarias para adaptarse a las necesidades y economía de cada institución.

\section{Limitaciones del estudio}

Existen dos aspectos principales que se encuentran fuera del alcance del presente estudio. En primer lugar, la revisión de herramientas se ha realizado conforme a las aplicaciones actualmente accesibles mediante búsqueda electrónica. Dado que este tipo de software permite una ventaja competitiva para clubes e instituciones deportivas, es probable que haya más herramientas de estas características que no hayan sido incluidas por haber sido creadas ad-hoc, de manera privada, y que, por lo tanto, no sean accesibles para su estudio en la actualidad. En segundo lugar, se hace necesario resaltar que, aunque se muestran las últimas tendencias comunes en las herramientas de gestión y evaluación del rendimiento, no se define un estándar de recomendaciones para implementar este tipo de aplicaciones.

\section{Conclusiones y aplicaciones prácticas}

La presente revisión refleja la progresiva tendencia de las organizaciones deportivas a gestionar el análisis del rendimiento y la planificación de los recursos demanera conjunta. Esta revisión pretende servir de ayudas tanto a científicos como técnicos deportivos en el progreso hacia un software más completo.

A pesar de esta tendencia, la mayoría de las herramientas aún están orientadas, bien al análisis del rendimiento, o bien, a la planificación de recursos. Sólo se han encontrado dos herramientas tecnológicas que integren ambos aspectos y las dos están creadas de manera específica para el fútbol.

En el dominio específico en que se encuadra este trabajo (baloncesto) no se han encontrado programas informáticos específicos que soporten la planificación y el análisis del rendimiento de las entidades deportivas de manera conjunta.

\section{Futuras líneas de investigación}

Tomando como referencia las herramientas desarrolladas para otros deportes, se propone investigar las siguientes cuestiones para desarrollar un sistema de estas características en baloncesto:

1) Realizar un análisis de presupuesto adaptado a la realidad de las entidades deportivas en el dominio del baloncesto.

2) Adaptar y mejorar el papel de los sistemas expertos de ayuda a toma de decisiones.

3) Desarrollar redes sociales integradas en la herramienta que permitan compartir conocimientos y fomentar el aprendizaje de tipo social.

4) Investigar sobre la correcta privacidad y confidencialidad de contenidos.
Baca,A., Dabnichki, P., Heller, M., \& Kornfeind, P. (2009). Ubiquitous computing in sports: A review and analysis. Journal of Sports Sciences, 27(12), 1335-1346. doi: 10.1080/02640410903277427 Campusdeportivovirtual. (2014). [software de computadora]. Bilbao: Campusdeportivovirtual, SL. Retrieved from http://e-ntrenamiento.com/home.asp

Cano, C. P., \& Cano, P. Q. (2006). Human resources management and its impact on innovation performance in companies. International Journal of Technology Management, 35(1-4), 11-28. doi: $10.1504 /$ ijtm.2006.009227

Dartfish. (2014). [software de computadora]. Fribourg Dartfish. Retrieved from http://www.dartfish.com DiamondScheduler. (2014). [software de computadora]. Cactusware. Retrieved from http:// www.cactusware.com/

Doherty, A. J. (1998). Managing Our Human Resources: AReview of Organisational Behaviour in Sport. Sport Management Review, 1, 1-24.

DVSport. (2014). [software de computadora]. Pittsburgh: DVSport, Inc. Retrieved from http:// dvsport.com/what-we-do/

Easy2Coach. (2014). [software de computadora]. Berlín: Easy2coach interactive. Retrieved from http://www.easy2coach.net/es/

Espor. (2014). [software de computadora]. Espor, Inc. Retrieved from http://www.espor.com/

Fernandez, J., Camerino, O., Anguera, M. T., \& Jonsson, G K. (2009). Identifying and analyzing the construction and effectiveness of offensive plays in basketball by using systematic observation. Behavior Research Methods, 41(3), 719-730. doi: 10.3758/brm.41.3.719

Franks, I. M., \& Goodman, D. (1986). A systematic approach to analysing sports performance. Journal of Sports Sciences, 4(1), 49-59. doi: 10.1080/02640418608732098

Gallardo-Guerrero, L., Garcia-Tascon, M., \& Burillo-Naranjo, P. (2008). New sports management software: A needs analysis by a panel of Spanish experts. International Journal of Information Management, 28(4), 235-245. doi: 10.1016/.ijinfomgt.2007.09-005

Gesdep.net. (2014). [sofware de computadora]. Las Palmas de Gran Canaria: Gestión Deportiva Consultores S.L. Retrieved from https://www.gesdep.net/web/

Grupo-campus. (2014). [software de computadora]. Bilbao: Grupo-campus. Retrieved from https:/ /sport-designer.com/

Hanisch, B., Hanisch, J., \& Pacis. (2007). Injury Management: The Development and Implementation of Innovative Software in an Elite Sporting Club. Kaohsiung: Natl Sun Yat-Sen Univ.

Hudl. (2014). [software de computadora]. Lincoln: Hudl. Retrieved from http://www.hudl.com/

Hughes, M. D., \& Franks, I. M. (2004). Notational analysis of sport: Better systems for improving coaching and performance (2nd ed.). London.

Hur, Y. J., Ko, Y. J., \& Valacich, J. (2011). A Structural Model of the Relationships Between Sport Website Quality, E-Satisfaction, and E-Loyalty. Journal of Sport Management, 25(5), 458-473.

Interplay-sports. (2014). [software de computadora]. Oslo: Interplay-sports. Retrieved from http:// www.interplay-sports.com/

JISC. (2014). Effective Assesment in a Digital Age. Retrieved from http://www.jisc.ac.uk/media/ documents/programmes/elearning/digiassass_eada.pdf

Kimetplanning. (2014). [software de computadora]. Getxo: Kimet Sport. Retrieved from http:// www.kimetsport.com/es

Kinovea. (2014). [software de computadora]. Kinovea. Retrieved from http://www.kinovea.org/

Li, C., \& Wang, Z. (2012). Research on the Applications of Information Technology in Sport Management. In X. Qu \& Y. Yang (Eds.), Information and Business Intelligence, Pt Ii (Vol. 268, pp. 247-252).

Liebermann, D. G., Katz, L., Hughes, M. D., Bartlett, R. M., McClements, J., \& Franks, I. M. (2002). Advances in the application of information technology to sport performance. Journal of Sports Sciences, 20(10), 755-769. doi: 10.1080/026404102320675611

Marcelo, J., \& Oltra, J. V. (2005). La mejora de la gestión del deporte adaptado mediante los sistemas de información. Revista de la Cámara de Comercio de Valencia, 2.

Markoski, B., Pecev, P., Ivkovic, M., Ivankovic, Z., \& Ratgeber, L. (2012). Applyment of basketball board for decision making in player management. Metalurgia International, 17(2), 100-109.

Mateos, L.A.(2011). SPXS Sports Picks eXpert System. Proceedings of the 2011 International Conference on Artificial Intelligence. (ICAI 2011). WORLDCOMP'11,320-325.

Mertz, L. (2013). Technology comes to the playing field: new world of sports promises fewer injuries, better performance. IEEE pulse, 4(5), 12-17. doi: 10.1109/mpul.2013.2271683

Misener, K., \& Doherty,A. (2009). ACase Study of Organizational Capacity in Nonprofit Community Sport. Journal of Sport Management, 23(4), 457-482.

Mundomonitor.com. (2014). [software de computadora]. Mundomonitor.com. Retrieved from http:/ /www.mundomonitor.com/

Nacsport. (2014). [software de computadora]. Las Palmas de Gran Canaria: Nacsport. Retrieved from http://www.nacsport.com/es

Nevill, A., Atkinson, G., \& Hughes, M. (2008). Twenty-five years of sport performance research in the Journal of Sports Sciences. Journal of Sports Sciences, 26(4), 413-426. doi: 10.1080/ 02640410701714589

NovaNET. (2014). [software de computadora]. Las Rozas: NOVANET, Automation Consultans. Retrieved from http://www.novanet.es/

Orekovic, B. (2007). Basketball logistic systems knowledge management. Osijek: Ekonomski Fakultet Osijeku-Fac Economics Osijek.

Procloud11. (2014). [software de computadora]. Málaga: Procloud11. Retrieved from http:// www.procloud11.com/

Rincoach. (2014). [software de computadora]. Las Rozas: Sportsulting, Management and Technology. Retrieved from http://www.rincoach.com/rc/

Schmidt, R. A., \& Lee, T. (1999). Motor control and learning. Champaifn, IL.: Human Kinetics.

Senlle, A., Gallardo, L., \& Dorado, A. (2004). Calidad en las organizaciones deportivas. Barcelona: Gestión 2000.

Simin, L., \& Jinhai, S. (2009). The construction of sports team training information platform based on browser/server and client/server. 2009 International Conference on Networking and Digital Society (ICNDS), 33-36. doi: 10.1109/icnds.2009.15

Skaut. (2014). [software de computadora]. Mutilva Alta: SKAUT Tech S.L. Retrieved from http:// www.skaut.es/es/Productos-de-software

SoccerLAB. (2014). [software de computadora]. Diepenbeek: SoccerLAB. Retrieved from http:// www.soccerlab.com/index.php

Sportstec. (2014). [software de computadora]. Warriewood: Sportstec ${ }^{\mathrm{TM}}$. Retrieved from http:// sportstec.com/

Travassos, B., Davids, K., Araujo, D., \& Esteves, P. T. (2013). Performance analysis in team sports: Advances from an Ecological Dynamics approach. International Journal of Performance Analysis in Sport, 13(1), 83-95.

Vidswap. (2014). [software de computadora]. VidSwap.com. Retrieved from http://vidswap.com/

Wu, H.-j., Zhao, H.-y., \& Zhao, J. (2013). Application of the cloud computing technology in the sports training. 20133rd International Conference on Consumer Electronics, Communications and Networks (CECNet), 162-165. doi: 10.1109/CECNet.2013.6703297 